\title{
Problemas de coexistencia entre Denominaciones de Origen Protegidas e Indicaciones Geográficas Protegidas: El caso "Aceite de Jaén"
}

\section{Introducción}

La provincia de Jaén se encuentra ubicada en el sureste de España, en la región (Comunidad Autónoma) de Andalucía, entre las provincias de Ciudad Real (norte), Granada (sur), Albacete (este) y Córdoba (oeste). Cuenta con una superficie de $13.489 \mathrm{~km}^{2}$, y una población de 654.170 habitantes (a 1 de enero de 2016), de los cuales aproximadamente una tercera parte viven en la capital (Jaén) y su área metropolitana. Administrativamente está conformada por 97 municipios agrupados en diez comarcas.

Con sus más de 500.000 hectáreas de olivar, es el mayor productor mundial de aceite de oliva, aportando más del 20 por ciento de la producción mundial de esta grasa vegetal. De ello se puede deducir la importancia que constituye este sector no sólo en la economía provincial, sino también en el ámbito social y cultural.

En la provincia existen tres Denominaciones de Origen para el aceite de oliva: DO Sierra de Segura, DO Sierra de Cazorla, y DO Sierra Mágina. Las Denominaciones de Origen, junto con las Indicaciones Geográficas, son referencias geográficas que nos proporcionan tanto información sobre el origen geográfico de un producto, como información sobre la presencia de especiales cualidades derivadas de ese origen, constituyendo un signo de calidad que relaciona el producto con su lugar de procedencia. Según la Organización Mundial de la Propiedad Intelectual (OMPI),

(p)or indicación geográfica se entiende un signo que se utiliza para productos de un origen geográfico específico y cuyas cualidades o reputación se deben a dicho lugar de origen. (...) Por lugar de origen se entiende una ciudad, un pueblo, una región, un país, etc. 
(...) Por denominación de origen, se entiende un tipo especial de indicación geográfica que se utiliza para productos que tienen cualidades específicas que se deben exclusiva o esencialmente al entorno geográfico de la elaboración del producto. En el concepto de indicación geográfica queda comprendido la denominación de origen. ${ }^{1}$

De esta definición podemos extraer que ambas figuras, indicaciones geográficas y denominaciones de origen, poseen dos funciones, digamos genuinas o esenciales: indicar el origen geográfico de un producto, por una parte, e informar sobre la presencia en el mismo de cualidades o características especiales que los diferencian de otros productos del mismo género, por otra. Pero, además de estas dos funciones principales, también estas figuras ejercen otras secundarias, como son la función de aglutinar la reputación o goodwill del producto o servicio, la función de distinguir la procedencia empresarial del mismo, así como la función de servir de instrumento de publicidad y promoción de los productos que identifican. ${ }^{2}$

Por otra parte, es característico de estas figuras la concurrencia de intereses públicos y privados. Entre los primeros, por un lado, nos encontramos con los intereses colectivos representados por los propios territorios, para los cuales las denominaciones de origen y las indicaciones geográficas suponen un importante motor de desarrollo socioeconómico. También a esta esfera pública pertenecen los intereses de los consumidores, a los cuales estas figuras proporcionan seguridad en el proceso de elección de un determinado producto que para ellos es portador de una calidad diferenciada y reduciendo la posibilidad de engaño. De otro lado, nos encontramos con los intereses de las empresas, que utilizan las indicaciones geográficas (en sentido amplio) como instrumentos de diferenciación de sus productos, beneficiándose de la atribución de cualidades superiores, lo que les reporta un mayor lucro y un mejor posicionamiento en los mercados. ${ }^{3}$ No obstante este interés privado, el carácter colectivo de las indicaciones geográficas permite que estos beneficios añadidos se compartan por un grupo de operadores y no a título individual como sucede en otras figuras de propiedad industrial. Los intereses públicos justifican la adopción de normas jurídico-públicas que pretenden el reconocimiento de las distintas indicaciones geográficas y la fijación de las características que deben reunir los productos bajo cuyo amparo se cobijan. Los intereses privados justifican la adopción de normas jurídico-privadas que configuran estas indicaciones como auténticos títulos de

${ }^{1}$ Organización Mundial de la Propiedad Intelectual (OMPI), Principios Básicos de la Propiedad Industrial, Publicación de la OMPI N 895(S), en: www.wipo.int, p. 16 y 17.

2 M.J. Botana Agra, Las Denominaciones de Origen, en: G. Jiménez Sánchez (coord.), Tratado de Derecho Mercantil, Vol. 2: Las Denominaciones de Origen, Barcelona 2001, pág. 36.

${ }^{3}$ M.J. Botana Agra, op. cit., p. 34 a 36. 
propiedad industrial, proporcionando un monopolio de uso que coincide con el de otros signos distintivos, como puede ser el de las marcas. ${ }^{4}$

Conjuntamente a esto, en las indicaciones geográficas, convienen dos concepciones o sistemas de protección. Un derecho represor de la competencia desleal, por un lado, frente a comportamientos de engaño, falsedad, confusión o aprovechamiento indebido de la reputación ajena, y en el que priman los intereses de los consumidores, conocido como sistema alemán. Y, por otro lado, un sistema de protección propia, que atribuye a las indicaciones geográficas un derecho de exclusiva o monopolio de utilización, en el cual se detecta la primacía de los intereses de los empresarios, conocido como sistema francés, y que progresivamente se va imponiendo en los foros internacionales y comunitarios. ${ }^{5}$

Como decimos, la existencia cada vez más intensa de productos con indicaciones relativas a su procedencia geográfica hace necesario el establecimiento de unos instrumentos de protección frente a comportamientos que atenten contra la veracidad de dicha procedencia, y esa protección, máxime conociendo el mercado global en el que nos manejamos, no puede limitarse al ámbito nacional, sino que debe alcanzar, cómo no, el ámbito comunitario e internacional.

La Comunidad Europea no es ajena a la importancia de estas figuras de calidad, razón por la que las ha regulado, originariamente con referencia a productos agroalimentarios, y está valorando en estos momentos la posibilidad de extender su protección hacia otros tipos de productos. Entre los productos protegidos por la actual normativa comunitaria de denominaciones de origen e indicaciones geográficas se encuentra el aceite de oliva. Como decíamos anteriormente, en la provincia de Jaén existen actualmente tres Denominaciones de Origen Protegidas (DOP) que amparan la fabricación de aceite de oliva, que se corresponden con tres comarcas naturales: Sierra Mágina, Sierra de Cazorla y Sierra de Segura, así como dos intentos fallidos para registrar otras dos denominaciones de origen para otras comarcas olivareras: DO "Campiñas de Jaén" y DO "Sierra Sur de Jaén". El rechazo de estas últimas por parte de la Comisión Europea, propició que se planteara el registro de una Indicación Geográfica Protegida (IGP) que aglutinara todos los aceites de oliva de calidad producidos en la provincia, decisión que chocó de lleno contra los intereses de las Denominaciones de Origen Protegidas preexistentes. El objeto de este trabajo es analizar este caso real de concurrencia de sendos tipos de figuras de protección en un mismo territorio para designar un mismo tipo de producto: el aceite de oliva virgen extra. El aceite de oliva virgen extra es un aceite de oliva virgen, es decir, el que se obtiene de la fábrica o almazara después de procedimientos exclusivamente físicos y/o mecánicos

${ }^{4}$ M.M. Gómez Lozano, Denominaciones de Origen y otras Indicaciones Geográficas, Navarra 2004, p. 31 y 32.

${ }^{5}$ M.J. Botana Agra, op.cit., p. 39 y 40. 
(molido, batido, prensado y/o centrifugación). Podemos decir que es un zumo de fruta, el zumo de la aceituna. El calificativo "extra" se otorga al aceite de oliva virgen que presenta unos resultados óptimos, tanto en el análisis químico como en el sensorial y solo se obtiene de aceitunas procedentes del árbol (nunca de aceitunas ya caídas al suelo). Es por tanto, un aceite sin defectos, el de mayor calidad que se puede producir, con una acidez inferior a $0,8^{\circ}$ y que ha cumplir con otros parámetros establecidos como índice de peróxidos, absorbancia en el ultravioleta, contenidos de ceras o ésteres alquílicos. ${ }^{6}$

\section{Objetivos y métodos}

El objetivo del presente trabajo es analizar un caso concreto y actual de concurrencia de Denominaciones de Origen Protegidas y de Indicaciones Geográficas Protegidas en un mismo territorio para designar un mismo producto, cual es el aceite de oliva virgen extra, caso que hemos denominado como caso "Aceite de Jaén"

Para ello, en primer lugar, se enumerarán los hechos acontecidos hasta la fecha en el devenir del caso, se analizará de una forma muy sucinta cada una de estas figuras de protección, DOP e IGP, para luego pasar a analizar las argumentaciones que las partes esgrimieron en defensa de sus intereses. A continuación, se analizará la resolución judicial firme, que arroja bastante luz al problema de la concurrencia de estas figuras y que pone un punto y seguido a un periplo que ya dura más de siete años y que se atisba que aún tenga que durar unos cuantos años más. Para concluir, expondremos los resultados de nuestra investigación y discutiremos sobre la conveniencia o no de la concurrencia de estas figuras de protección en un mismo territorio.

\section{Resultados y discusión}

La Federación de Productores de Aceite de Oliva de Jaén presentó solicitud de inscripción de la Indicación Geográfica Protegida "Aceite de Jaén" en el correspondiente registro comunitario, publicándose en el BOE de 4 de agosto de 2010 el anuncio de dicha solicitud por parte de la Dirección General de Industrias y Calidad Agroalimentaria de la Consejería de Agricultura y Pesca de la Junta de Andalucía, en virtud de Resolución de 25 de junio de 2010.

${ }^{6}$ F. Palomeque Messía, F.R. Palomeque Ruiz, F. Montes Tubío, E. Burgos Ladrón de Guevara, Tipos de Aceite de Oliva, en: (Coords), El Olivar y su Aceite. F. Palomeque Messía, A. Martínez Gutiérrez, J.L. Vílchez Quero y G. Rodríguez Navarrete, Fundación del Olivar, 2013, págs. 124 y 125. 
Dentro del plazo legal, con fecha 4 de octubre de 2010, el Consejo Regulador de la Denominación de Origen Protegida "Sierra de Cazorla" y la "Asociación Empresarial Agronómica para la mejora de la calidad del Aceite de Oliva Sierra de Cazorla" presentaron oposición a dicha solicitud de inscripción. No obstante la oposición, la Consejería de Agricultura y Pesca de la Junta de Andalucía, a través de la Orden de 10 de diciembre de 2010, emitió decisión favorable en relación con la solicitud de inscripción de la Indicación Geográfica Protegida “Aceite de Jaén", publicándose la misma en el BOJA núm. 246, de 20 de diciembre de 2010.

Contra la referida Orden, el Consejo Regulador de la Denominación de Origen "Sierra de Cazorla" presentó recurso de reposición, el cual fue desestimado por Resolución de la Consejería de Agricultura y Pesca, de 2 de marzo de 2011.

La solicitud de inscripción de dicha IGP fue transmitida en fecha 8 de marzo de 2011 a la Comisión Europea, publicándose en el BOE de 15 de junio de 2011, por parte del Ministerio de Medio Ambiente, y Medio Rural y Marino, mediante Resolución de 27 de mayo de 2011 de la Dirección General de Industria y Mercados Alimentarios, el pliego de condiciones de la referida IGP, a efectos de la protección nacional transitoria prevista en el artículo 5.6 del Reglamento (CE) 510/2006, cuya petición había sido remitida a dicho Ministerio por parte de la Dirección General de Industrias y Calidad Agroalimentaria de la Consejería de Agricultura y Pesca de la Junta de Andalucía.

El día 12 de mayo de 2011, la "Asociación Empresarial Agronómica para la mejora de la calidad del Aceite de Oliva Sierra de Cazorla" presentó Recurso Contencioso-Administrativo ante el Tribunal Superior de Justicia de Andalucía contra la Resolución de fecha 2 de marzo de 2011 dictada por la Consejería de Agricultura y Pesca de la Junta de Andalucía, que desestima el recurso de reposición interpuesto el día 19 de enero de 2011 por la entidad recurrente contra la Orden de la Consejería de Agricultura y Pesca de 10 de diciembre de 2010, por la que se emite declaración favorable en relación con la solicitud de inscripción de la Indicación Geográfica Protegida „Aceite de Jaén”.

El 25 de octubre de 2011, la Sala de lo Contencioso-Administrativo del Tribunal Superior de Justicia de Andalucía dictó Auto por el cual se ordena suspender cautelarmente la tramitación administrativa de la inscripción de la IGP "Aceite de Jaén" en el registro comunitario de denominaciones de origen protegidas e indicaciones geográficas protegidas. Dicho Auto fue confirmado por Auto de 22 de noviembre de 2011.

En virtud de dicho Auto, en fecha 19 de diciembre de 2011 (BOE de 9 de enero de 2012) la Dirección General de Industria y Mercados Alimentarios del Ministerio de Medio Ambiente, y Medio Rural y Marino, emitió Resolución por la que se deja en suspenso la protección nacional transitoria concedida a la IGP 
“Aceite de Jaén", comunicando a la Comisión Europea la existencia del citado recurso contencioso-administrativo y la suspensión cautelar del procedimiento acordada por el Tribunal.

El 23 de mayo de 2016, cinco años después de presentarse el recurso ante el Tribunal Superior de Justicia de Andalucía, la Sala de lo Contencioso Administrativo de dicho Tribunal, a través de la Sentencia número 1.489 de 2016, falló a favor de la Consejería de Agricultura y Pesca de la Junta de Andalucía, desestimando el recurso contencioso-administrativo interpuesto por la "Asociación Empresarial Agronómica para la Mejora de la Calidad del Aceite de Oliva en la Sierra de Cazorla", que adquiere firmeza al no presentarse recurso de casación ante el Tribunal Supremo, lo que deja abierto el cauce para una posible nueva solicitud de inscripción de la IGP "Aceite de Jaén" ante las instancias europeas por parte de sus promotores. ${ }^{7}$

Enumerados los hechos acontecidos, pasaremos a continuación a analizar el caso desde el punto de vista jurídico.

En primer lugar, y a efectos de delimitación, hay que tener claro que el producto amparado tanto por la DOP "Sierra de Cazorla", como por la pretendida IGP "Aceite de Jaén" es el aceite de oliva virgen extra, principalmente de la variedad picual. Por otra parte, la zona geográfica delimitada por la citada IGP abarca toda la provincia de Jaén, mientras que la zona delimitada por la DOP engloba los términos municipales de Cazorla, Chilluévar, Hinojares, Huesa, La Iruela, Peal de Becerro, Pozo Alcón, Quesada y Santo Tomé, de la provincia de Jaén. Por tanto, IGP y DOP coinciden en el territorio de esta última, solapándose ambas figuras de protección en la citada área para designar un mismo producto, el aceite de oliva virgen extra.

La oposición planteada por el Consejo Regulador de la Denominación de Origen Protegida "Sierra de Cazorla" y por la "Asociación Empresarial Agronómica para la Mejora de la Calidad del Aceite de Oliva en la Sierra de Cazorla" contra la solicitud de inscripción de la IGP "Aceite de Jaén" en el registro comunitario, y que fundamentalmente constituyó la base del recurso contencioso-administrativo posterior, se sustanció en las causas de oposición contenidas en las tres primeras letras del artículo 7 del Real Decreto 1069/2007, de 27 de Julio, por el que se regula el procedimiento para la tramitación de las solicitudes de inscripción en el Registro comunitario de las denominaciones de Origen Protegidas y de las Indicaciones Geográficas Protegidas y la oposición a ellas.

7 Sentencia del Tribunal Superior de Justicia de Andalucía. Sala de lo Contencioso-Administrativo. Sede Granada. Sección Primera. Recurso No 1318/2011. Sentencia Núm. 1.489 de 2016. (Roj: STSJ AND 5207/2016 - ECLI:ES:TSJAND:2016:5207). Puede consultarse en: http://www. poderjudicial.es. 
Según este artículo, “(...) la declaración de oposición sólo será estimada si:

a) Demuestra el incumplimiento de las condiciones que deben reunir las denominaciones de origen y las indicaciones geográficas para ser consideradas como tales, de acuerdo con el artículo 2 del Reglamento (CE) n ${ }^{0}$ 510/2006 del Consejo, de 20 de marzo.

b) Demuestra que el registro del nombre propuesto podría inducir a error al consumidor por entrar en conflicto con el nombre de una variedad vegetal o de una raza animal, existir una denominación total o parcialmente homónima o afectar a la reputación de una marca, de acuerdo con el artículo 3, apartados 2, 3 y 4, del citado Reglamento.

c) Demuestra que el registro del nombre pondría en peligro la existencia de una denominación total o parcialmente homónima o de una marca registrada o la existencia de productos que se hayan estado comercializando legalmente durante al menos los cinco años anteriores a la fecha de publicación en el "Boletín Oficial del Estado" de la solicitud de inscripción y del Documento único.

d) Demuestra que el nombre cuyo registro se solicita tiene carácter genérico por haber pasado a ser el nombre común de un producto agrario o alimenticio, según lo dispuesto en el artículo 3.1 del Reglamento (CE) n n 510/2006 del Consejo, de 20 de marzo."

Como hemos dicho anteriormente, los motivos de oposición se basaron en la concurrencia de las tres primeras causas contenidas en el artículo 7 del citado Real Decreto.

Con relación a la primera causa, incumplimiento de las condiciones que deben reunir las DOP e IGP para ser consideradas como tales, debemos recordar el artículo 2 del Reglamento 510/2006, el cual definía los conceptos de DO e IG. Para cualquiera de los dos figuras de protección, se trata "(d)el nombre de una región, de un lugar determinado o, en casos excepcionales, de un país, que sirve para designar un producto agrícola o un producto alimenticio originario de dicha región, de dicho lugar determinado o de dicho país (...)".

Como indicábamos anteriormente, las diferencias entre denominaciones e indicaciones residen en la intensidad del doble vínculo cualitativo y geográfico, mucho más marcado en las primeras que en las segundas. Con relación al vínculo cualitativo; mientras que para las indicaciones es suficiente que cualquier cualidad o característica del producto pueda ser atribuible al origen geográfico; para las denominaciones es preciso que la "calidad o características del producto se deban fundamental o exclusivamente al medio geográfico con sus factores naturales y humanos". Respecto al vínculo geográfico, para las indicaciones basta con que cualquier fase del proceso productivo (producción, transformación o elaboración) se realice en la zona, mientras que en las denominaciones es obligatorio que todas estas fases radiquen en el área geográfica delimitada. 
Con relación a este punto, en fase administrativa, la parte opositora alegó que tras un análisis del pliego de condiciones de la IGP "Aceite de Jaén", se podía observar que la vinculación entre producto y territorio era total, correspondiendo más a una vinculación propia de una DO que de una IG. En palabras de los opositores, "nos encontramos ante una solicitud de DO bajo, no obstante, el nomen iuris de IGP".

Esta tesis fue rechazada por la Junta de Andalucía, argumentando que la exigencia de una mayor calidad y vinculación al territorio por parte de la controvertida IGP no puede ser una causa de oposición a la misma, sino más bien "un aval en su reconocimiento como IGP". Por su parte, el TSJ, en su resolución al recurso contencioso-administrativo, con relación a esta causa de oposición, rechaza igualmente el argumento de la parte actora, concluyendo que en este caso se dan los requisitos que marca la normativa comunitaria para la existencia de una IGP y que no hay nada que objetar a que un mismo producto pudiera tener encaje en ambas figuras de protección, no señalando la normativa que deba darse prioridad a una figura sobre otra, y, añadiendo, que de la lectura del pliego de condiciones, las características de la IGP “Aceite de Jaén” se centran más en las condiciones del medio geográfico que en sus factores humanos.

No obstante, si analizamos el pliego de condiciones de la IGP "Aceite de Jaén", podemos observar que la doble vinculación cualitativa y geográfica entre producto y territorio es total, correspondiendo más a una vinculación propia de una DO que a una IG. En este sentido, el punto 5 del citado pliego de condiciones determina: "Las fases de producción y elaboración, de los aceites amparados bajo la Indicación Geográfica, se realiza en la zona delimitada". Y en el párrafo anterior afirma: "Se establecerá un sistema de trazabilidad que garantice que el origen, la elaboración y condiciones del producto procede de la zona delimitada por este pliego de condiciones".

En cuanto a los parámetros de calidad, es curioso que para los aceites amparados por la IGP las exigencias sean más elevadas que para los amparados por la DOP. Valgan como ejemplos el grado de acidez máximo admitido de $0,5 \%$ para los productos amparados por la IGP “Aceites de Jaén", frente al máximo de 0,7\% de la DOP "Sierra de Cazorla"; el índice de peróxidos máximo de 15, del primero, frente al 20 del segundo, o el índice de absorbancia en el ultravioleta (coeficiente K270), superior en la DOP y, por tanto, indicativo de una menor capacidad antioxidante del aceite, que en la IGP.

Efectivamente, la normativa comunitaria no determina que se establezca un criterio de prioridad de una figura sobre otra, pero si analizamos estas figuras a la luz de la normativa internacional, vemos claramente que la DO es un tipo especial de IG. Si admitiéramos mayores exigencias a una IG que a una DO, nos encontraríamos con la extraña situación de que un producto amparado por 
la primera no pudiera estarlo por la segunda, o, lo que trasladado a nuestro caso sería, que un aceite amparado por la DOP "Sierra de Cazorla" podría no estarlo bajo la IGP "Aceite de Jaén". Por tanto, sería inaplicable la afirmación que realiza el TSJ de que un mismo producto pudiera tener encaje en ambas figuras.

La segunda causa de oposición versó sobre la posibilidad de que el nombre propuesto pudiera inducir a error al consumidor por entrar en conflicto con el nombre de una variedad vegetal o de una raza animal, existir una denominación total o parcialmente homónima o afectar a la reputación de una marca, de acuerdo con el artículo 3, apartados 2, 3 y 4 del Reglamento (CE) 510/2006.

Este segundo supuesto se fundamenta en la mera posibilidad (no necesariamente certeza) de que se induzca a error al consumidor en cualquier de estos tres supuestos:

1. Cuando el nombre propuesto entre en conflicto con el nombre de una variedad vegetal o de una raza animal y pueda inducir a error en cuanto al verdadero origen del producto.

2. En el supuesto de homonimia total o parcial, cuando se induzca a creer erróneamente que los productos son originarios de otro territorio.

3. Cuando habida cuenta de la reputación de una marca, su notoriedad y la duración de su uso, se pueda inducir a error al consumidor en cuanto a la verdadera identidad del producto.

En el caso que aquí nos compete, obviamente no se produce el primero de estos tres supuestos, ya que la denominación o, en este caso, indicación geográfica "Aceite de Jaén" no entra en conflicto con ninguna variedad vegetal o raza animal, siendo el aceite un producto obtenido del fruto del olivo (olea europea), una variedad vegetal muy extendida en los países mediterráneos y que no es originaria de una zona exclusiva o delimitada.

Con relación al segundo supuesto, la Junta de Andalucía, se limita a decir que "Sierra de Cazorla" no presenta similitud alguna con "Aceite de Jaén". El TSJ, a esta afirmación, añade que "se trata de dos signos de calidad que operan en distintos niveles (...) que divergen en su ámbito territorial". No podemos compartir ninguno de estos criterios.

Es cierto que, aparentemente, no existe homonimia alguna entre las denominaciones "Sierra de Cazorla" y "Aceite de Jaén". Sin embargo, para apreciar el riesgo de error en el consumidor, que es de lo que se trata, hay que tener en cuenta no solo las estructuras de ambas denominaciones, sino la evocación o sugestión que provocan en el consumidor. En este sentido, nos encontramos, por un lado, con una denominación sugestiva, "Sierra de Cazorla". Se trata de un nombre de una zona geográfica concreta, parte integrante de un parque natural de la provincia de Jaén, que aplicado a un producto, como es el aceite, evoca en el consumidor el origen natural de un producto proveniente de esta famosa sierra 
de Jaén. Por otro lado, nos encontramos con una denominación parcialmente descriptiva, parcialmente sugestiva, cual es "Aceite de Jaén". Esta denominación nos describe el producto amparado por la misma, el aceite, y mediante la adición del término "Jaén" pretende sugerir al consumidor que el mismo posee una serie de cualidades que la reputación de esta tierra ha ido consagrando a lo largo del tiempo. Pues bien, desde nuestro punto de vista, entendemos que si el consumidor identifica el término "Sierra de Cazorla" con el término "Jaén", cuando ambos términos los aplicamos a un mismo producto, como es el aceite, es más que posible que se induzca a error en el consumidor sobre el verdadero origen de uno y otro producto.

En apoyo de esta tesis, debemos mencionar las resoluciones judiciales que solucionaron el conflicto entre la Denominación de Origen "Jamón de Huelva" y la denominación social "Origen Jabugo", para identificar productos derivados del cerdo (jamones, paletillas y otros). La Audiencia Provincial de Huelva, en Sentencias de 8 y 20 de febrero, afirmó que “(...) tratándose del comercio del mismo género de productos y siendo que la denominación de origen que administra la actora resulta ser más amplia y englobar la zona mencionada en el nombre discutido, es evidente la competitividad entre uno y otro sello de calidad; y de ello se deduce que la entidad demandada se acercó a los linderos de lo dudoso haciendo uso como signo de identificación de sus productos de una denominación social que estaba - al menos en mayor medida que otros posibles nombres- en los límites de lo admisible cuando se emplea como equivalente a una marca (...)"8

Por otra parte, ¿cómo puede afirmar el TSJ que se trata de dos signos de calidad que operan a distintos niveles y que divergen en su ámbito territorial? ¿Acaso el territorio de la DO "Sierra de Cazorla" no está dentro del de la IG "Aceite de Jaén"? ¿No sería más acertado afirmar que existe una convergencia territorial coincidente con el territorio de la DO? ¿Y qué es eso de que operan a distintos niveles? Ambos signos distintivos van a operar en los mismos mercados, a nivel local, nacional e internacional, siendo competencia directa a todos los niveles.

La segunda causa de oposición presentada atiende también al supuesto de inducción a error en cuanto a la verdadera identidad del producto, habida cuenta de la reputación de una marca, su notoriedad y la duración de su uso. Para la Junta de Andalucía, al no estar hablando de una marca, sino de una DOP, no debe aplicarse el precepto. El TSJ no se pronuncia sobre la extensión o no del precepto a otros signos distintivos diferentes de las marcas, reiterando que no se induce a error en el consumidor sobre la identidad de los signos.

${ }^{8}$ Sentencia de la Audiencia Provincial de Huelva, de 8 de febrero de 2006, Fundamento de Derecho Segundo, apartado B. (Roj: SAP H 242/2006). Puede consultarse en: http://www. poderjudicial.es. 
A este respecto, podemos apuntar que, si bien el precepto hace referencia al signo distintivo por excelencia, la marca, sería sensato hacer extensiva la prohibición de registro frente a la preexistencia de cualquier otro signo distintivo, diferente de una marca, que goce de la mencionada reputación, notoriedad y duración de uso, cual es el caso de una denominación de origen.

En cuanto a la inducción a error en el consumidor, la reputación, notoriedad y duración de uso están más que demostradas en el caso de la Denominación de Origen "Sierra de Cazorla", pudiendo a nuestro juicio generar error en el consumidor a la hora de identificar un producto amparado bajo el sello de esta denominación preexistente y de la pretendida IGP "Aceite de Jaén", habida cuenta de las importantes conexiones existentes entre ambas denominaciones, tal como hemos expresado anteriormente y por el hecho de la existencia de coincidencias territoriales entre las mismas, que en el caso de la IGP abarca la totalidad del ámbito geográfico de la DOP.

La tercera causa de oposición, como indicábamos, se sustenta en la letra c) del artículo 7 del Real Decreto 1069/2007, es decir, en la puesta en peligro de la existencia "de una denominación total o parcialmente homónima o de una marca registrada o la existencia de productos que se hayan estado comercializando legalmente durante al menos los cinco años anteriores a la fecha de publicación en el "Boletín Oficial del Estado" de la solicitud de inscripción y del Documento único".

Para combatir esta causa de oposición, la Junta de Andalucía se hace eco de un informe de la Dirección General de Agricultura y Desarrollo Rural de la Comisión Europea, el cual no referencia, y que habla de la posibilidad de que una zona de producción de un nombre registrado pueda solaparse con la de otro o la incluya totalmente, en el caso de productos similares. En estos casos, para poder comercializarse con ambas menciones se debe cumplir con los requisitos y sujetarse al régimen de control establecidos en el pliego de condiciones de cada una de ellas, siempre y cuando el etiquetado evite inducir a error a los consumidores. El TSJ reproduce el mencionado informe de la Dirección General y concluye: "se trata de dos títulos plenamente compatibles, no contrapuestos. Habrá productos que reúnan únicamente las condiciones de la IGP „Aceite de Jaén”, y otros en los que concurran los requisitos de ambos para poder incluir en su etiquetado tanto la IGP „Aceite de Jaén” como la DO „Sierra de Cazorla”.” Con ello el Tribunal parece admitir la posibilidad lógica del doble etiquetado IGP-DOP para los productos ya amparados por la DOP, pero ¿qué ocurrirá con aquellos aceites que ya operan bajo la DOP que no cumplen con los requisitos de la IGP? Según este criterio no podrían acceder al doble etiquetado y, por tanto, no podrían compatibilizar los títulos. Es decir, para esos aceites ambos signos serían contrapuestos y, por tanto, rivales. 
Hecha esta indicación, deberíamos analizar, por tanto, en primer lugar, si la IGP "Aceite de Jaén" pondría en peligro la existencia de la Denominación de Origen "Sierra de Cazorla".

Para el TSJ está claro al desestimar este motivo de oposición presentado por la parte actora cuando concluye: "no ha quedado suficientemente acreditado que la inscripción de la IGP „Aceite de Jaén” implique un peligro para la existencia de la DO „Sierra de Cazorla”", aludiendo a la, a su juicio, plena compatibilidad y no contraposición de ambos títulos antes mencionada y que ya hemos visto que hace aguas.

Es más, para conocer este peligro sería preciso conocer cómo se sustenta la mencionada Denominación de Origen. Si acudimos al Reglamento de la Denominación de Origen Sierra de Cazorla y de su Consejo Regulador, aprobado por Orden de la Consejería de Agricultura y Pesca de la Junta de Andalucía, de 9 de noviembre de 2000 (BOJA núm. 138, de 30 de noviembre), podemos leer en su artículo 20, punto 1: "Sólo las personas físicas o jurídicas que tengan inscritos sus olivares, almazaras o plantas envasadoras-comercializadoras en los Registros a que se refiere el artículo 14 podrán, respectivamente, producir aceituna con destino a la elaboración de aceites protegidos, o molturar dicha aceituna y obtener aceite con derecho a la Denominación de Origen, o envasar aceites protegidos por la Denominación." Y el punto 4 continúa: "Por el mero hecho de la inscripción en los Registros correspondientes, las personas físicas o jurídicas inscritas quedan obligadas al cumplimiento de las disposiciones de este Reglamento y de las que dentro de sus competencias dicten el Ministerio de Agricultura, Pesca y Alimentación y la Consejería de Agricultura y Pesca de la Junta de Andalucía, así como a satisfacer las tasas que les correspondan."

Continuando con la lectura del Reglamento, nos encontramos con el artículo 43, el cual habla de la financiación de las obligaciones del Consejo Regulador y enumera los recursos con los que se efectuarán, mencionando entre ellos y en primer lugar la cantidad recaudada de la aplicación de tasas, estableciendo las siguientes:

a) Tasa anual sobre las plantaciones inscritas, que se aplica en función del número de hectáreas inscritas por cada interesado.

b) Tasa sobre los productos amparados, basada en el valor de las facturas realizadas por la venta del aceite protegido.

c) Tasa por derecho de expedición de cada certificado de origen, visado de factura, compulsa y venta de precintos y contraetiquetas, en función de su precio de coste.

En definitiva, gran parte del organigrama que sustenta la Denominación de Origen "Sierra de Cazorla" se basa en el cobro de unas tasas que deben satisfacer los titulares inscritos en los registros de la Denominación. Pues bien, la introduc- 
ción de una IGP, como la de "Aceite de Jaén", para amparar un mismo producto, cual es el aceite de oliva virgen extra, y en el mismo territorio que abarca la DOP "Sierra de Cazorla", genera competencia directa sobre esta última, ejerciendo atracción sobre agricultores, productores, envasadores y comercializadores de la zona, los cuales pueden ver como mejor opción integrarse en la nueva IGP, en detrimento de la DOP, o, incluso, abandonarla, bien por asumir unos costos más bajos, bien por otras cuestiones como mejor promoción, posicionamiento, etc., lo cual pondría en serio peligro la existencia de la DOP. Pero es que, además, las numerosas marcas registradas que comercializan actualmente sus productos bajo el paraguas de la Denominación de Origen "Sierra de Cazorla" podrían ver reducidas sus ventas y con ellas, peligrar igualmente su existencia, en el caso de que otras marcas competidoras eligieran la cobertura de la nueva IGP “Aceite de Jaén" o, incluso, verse forzadas a incluir un doble etiquetado en el que aparezcan ambos signos distintivos, al objeto de no distanciarse de estos nuevos competidores, con el consiguiente aumento de costes provenientes del duplicado de tasas. Concluyendo, al contrario de lo que opinan Junta de Andalucía y TSJ, la IGP “Aceite de Jaén", tal y como está planteada, pone en serio peligro la continuidad de la DOP "Sierra de Cazorla".

Resultados de le investigación y la discusión

Tras cinco años de espera, el Tribunal Superior de Justicia de Andalucía se ha pronunciado sobre el fondo del asunto, rechazando todos los motivos de oposición a la solicitud de inscripción de la IGP “Aceite de Jaén”. Esto, sin duda, va a motivar a los promotores aspirantes a este nuevo título a presentar de nuevo su solicitud de registro para su tramitación por la Comisión Europea. No obstante este pronunciamiento, desde nuestro criterio, creemos que las oposiciones presentadas contenían fundamentos suficientes para haber rechazado la pretensión de acceso al registro de esta nueva IGP "Aceite de Jaén". Ello es así porque, como hemos tenido oportunidad de argumentar, en primer lugar existe un error a la hora de conceptualizar la IGP, la cual se ha configurado más sobre los criterios de una DOP que sobre los de una IGP, no dejando margen para la coexistencia de otras realidades enraizadas ya en el territorio, como las diferentes denominaciones de origen preexistentes, pues les impide a éstas la inclusión en la nueva IGP, marcándoles unos criterios de calidad superiores que los establecidos para dichas denominaciones. En segundo lugar, con la puesta en marcha de la nueva IGP creemos que se corre el riesgo de inducir a error en el consumidor, tanto sobre el origen del producto, como sobre la identidad del mismo, puesto que coexistiría con una denominación que evocaría en la mente del consumidor un mismo lugar, y por verse además afectada la reputación de un signo distintivo preexistente. Por último, es clara la puesta en peligro de la existencia de una denominación anterior con la que guarda una evidente similitud y de los productos comercializados bajo 
su amparo durante un período de tiempo oportuno, al entrar en directa competencia los productos designados por uno y otro título.

El presente caso es un claro ejemplo de las dificultades que presenta la coexistencia o concurrencia de una DOP y una IGP en un mismo territorio para designar una misma clase de productos, y la convivencia entre ambas figuras no debería admitirse desde nuestro punto de vista, ya que, al contrario de lo que opina el TSJ, entendemos que ambos títulos se excluyen mutuamente, puesto que, tal y como está planteado el pliego de condiciones de la IGP, los productos amparados por la DOP no van a estar necesariamente protegidos también por la IGP.

Entendemos que sería muy positivo para un mejor posicionamiento en los mercados y una mejor promoción de los diferentes productos que conviven en la provincia de Jaén contar con un título de calidad que los aglutine a todos ellos a escala provincial. Pero esta noble pretensión no debería llevarse a cabo a costa de la desaparición de otros títulos preexistentes más que consolidados, que llevan varias décadas realizando una importante labor y que han contribuido a la producción de un aceite de mejor calidad, a la creación de una mejor imagen del mismo en los mercados y, sobre todo, que han generado un mayor valor añadido que ha repercutido directamente en productores, agricultores y en la sociedad de la comarca en su conjunto. Sería deseable, por tanto, que este nuevo título de calidad, la IGP “Aceite de Jaén", supiera respetar estos títulos preexistentes, y que los productos amparados por las tres denominaciones de origen de la provincia pudieran acceder sin dificultades a la nueva IGP, para poder contar con ese doble etiquetado desde un primer momento, bien exonerándoles del cumplimiento de esos criterios de calidad más exigentes, bien estableciendo para ellos unos períodos de adaptación realizables. Asimismo, sería loable establecer para ellos unas tasas más reducidas para conseguir la pertenencia a la IGP, al objeto de que el esfuerzo económico de los productores no se viera duplicado por pertenecer a ambas. También sería oportuno establecer la exigencia a todos los aceites elaborados en el territorio de cualquiera de las tres DO de pertenecer necesariamente a éstas para poder acceder a la protección de la IGP. De este modo, las Denominaciones de Origen Protegidas no tendrían que competir en su territorio con aceites amparados por otros títulos de calidad que pusieran en entredicho su propia calidad, pues el consumidor no es consciente de las leves diferencias existentes entre una DOP y una IGP. En definitiva, no debería admitirse que un producto amparado por una de las tres DOP no lo estuviera a su vez por la IGP "Aceite de Jaén", y, por otro, no debería permitirse que un aceite elaborado en el territorio de una DOP pudiera pertenecer a la IGP sin pertenecer a su vez a la DOP. De otro modo, la pervivencia de las denominaciones preexistentes y la convivencia de las mismas con la nueva IGP sería imposible, configurándose más como feroces competidores que como aliados en aras de un objetivo común 
y colectivo, cual debería ser el proporcionar un mejor posicionamiento en los mercados de los aceites de calidad elaborados en nuestra provincia, y no servir únicamente para satisfacer los intereses particulares de algunos productores con poca mentalidad colectiva.

\title{
PROBLEMS OF THE CO-EXISTENCE OF THE PROTECTED DESIGNATIONS OF ORIGIN AND PROTECTED GEOGRAPHICAL INDICATIONS: THE CASE “ACEITE DE JAÉN"
}

\section{Sum mary}

Designations of Origin and Geographical Indications are two quality titles covered by EU legislation for the protection of agricultural products and foodstuffs whose quality is linked to a specific territory. In spite of being two different titles, the similarities between them are evident, and, since the EU regulations do not establish priority or incompatibility between them, it is possible that both protection instruments can apply in the same territory to designate the same type of product. This is actually the case in the province of Jaén, in southeastern Spain, where three Protected Denominations of Origin (PDOs) are threatened as a result of the application for registration of a Protected Geographical Indication (PDI) that intends to act at the provincial level for designating the same product: extra virgin olive oil. In this study we analyse this case and formulate some general recommendations of possible solutions that might allow a peaceful coexistence between these two titles of protection.

\section{PROBLEMI DI COESISTENZA DEI MARCHI DI DENOMINAZIONE DI ORIGINE PROTETTA E INDICAZIONE GEOGRAFICA PROTETTA: IL CASO DELL'«OLIO D’OLIVA DI JAÉN»}

\author{
Riassunto
}

I marchi Indicazione Geografica Protetta e Denominazione di Origine Protetta sono stati previsti nella legislazione dell'UE per tutelare i prodotti agroalimentari certificando la loro peculiare qualità, tipica di una determinata area geografica. Nonostante si tratti di due diverse forme di tutela giuridica, esse presentano molti tratti simili. Va sottolineato che nei regolamenti dell'UE non è stato determinato un ordine di gerarchia nel ricorrere a queste forme di tutela, così come non sono state determinate le circostanze del conflitto di coesistenza. Ciò significa che gli imprenditori possono beneficiare di entrambi i marchi per tutelare la qualità dei prodotti agroalimentari allo stesso tempo. Un tale problema è stato riscontrato nella provincia di Jaén, al sud della Spagna, dove il ricorso a tre Indicazioni Geografiche Protette è stato messo in dubbio con la presentazione di una domanda di registrazione della Denominazione di Origine Controllata riferita allo stesso prodotto - l'olio extravergine d'oliva di prima spremitura. A sostenere la tesi di una possibile coesistenza di queste due forme di tutela giuridica in un determinato territorio, l'Autore ha presentato le posizioni emerse nella dottrina e nella giurisprudenza, supportate da riflessione individuale. 
